\title{
Impacto sobre el índice de masa corporal en niños con leucemia aguda linfoide apoyados o no con un plan alimentario en el Centenario Hospital Miguel Hidalgo de la ciudad de Aguascalientes
}

Serrano-López Mario Alberto*, Rubalcava-Rodríguez Martha*, Galindo-López Claudia*, Bernal-Macías Karla Patricia*, Serna $B^{*}$, De Anda-de Lira Karina*, Pérez-Maldonado Ricardo**, Rosas-Cabral Alejandro***.

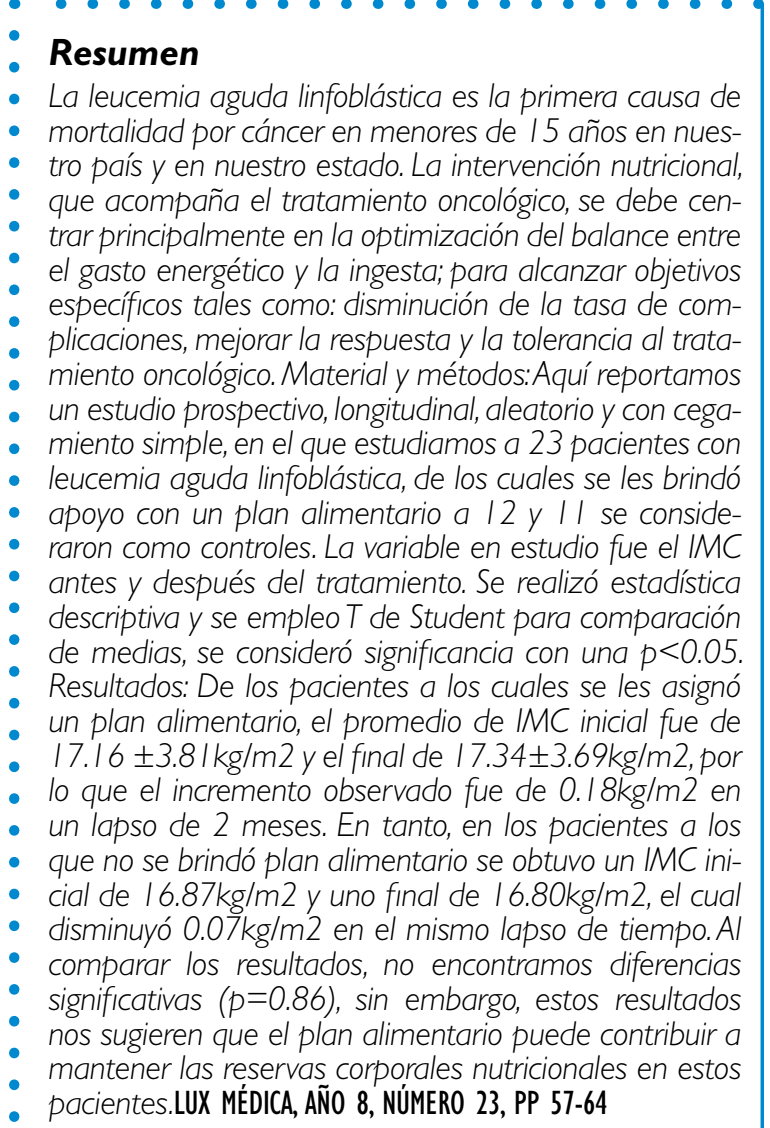

- Palabras clave: leucemia aguda linfoblástica, índice de masa corporal, intervención nutricional, niños

\section{Abstract}

Acute lymphoblastic leukemia is the main cause of cancer death in children under 15 years in our country and in our state. Nutritional intervention, that accompanies cancer treatment, should mainly focus on optimizing the balance between energy expenditure and ingest; to achieve specific objectives such as: decrease in the rate of complications, improve response and tolerance to cancer treatment. Material and methods: We report a prospective, longitudinal, randomized, single blinded, study in which were studied 23 patients with acute lymphoblastic leukemia, which has provided support with a meal plan to 12 and I I were considered as controls. The variable in study was the body mass index (BMI) before and after the treatment. Descriptive statistics, and Student's T was employed for comparison of means was considered significance with a $p<$ 0.05. Results: Patients were assigned to which a meal plan, the initial BMl average was $17.16 \pm 3.81 \mathrm{~kg} / \mathrm{m} 2$ and the end of $17.34 \pm 3.69 \mathrm{~kg} / \mathrm{m} 2$, so the observed increase was $0.18 \mathrm{~kg} / \mathrm{m} 2$ in a period of two months. Meanwhile, in patients not provided that meal plan was obtained an initial BMl of $16.87 \mathrm{~kg} / \mathrm{m} 2$ and the end of $16.80 \mathrm{~kg} / \mathrm{m} 2$, which decreased $0.07 \mathrm{~kg} / \mathrm{m} 2$ in the same period of time. To compare the results, found no significant differences ( $p=0.86$ ), however, these results suggest us that the meal plan can help maintain nutritional body reserves in these patients. LUX MÉDICA, AÑO 8, NÚMERO 23, PP 57-64

Key words: leukemia acute lymphoblastic, (BMI) Body Mass Index, nutritional intervention

* Departamento de Nutrición, Cultura Física y Deporte. Universidad Autónoma de Aguascalientes

** Servicio de Oncología Pediátrica. Centenario Hospital Miguel Hidalgo

*** Departamento de Medicina. Universidad Autónoma de Aguascalientes

Fecha de recibido: 10 de enero 2013

Fecha de aceptación: 30 de enero 2013

Correspondencia

Rosas-Cabral Alejandro. Departamento de Medicina del Centro de Ciencias de la Salud de la Universidad Autónoma de Aguascalientes. Av. Universidad \#940 Ciudad Universitaria CP 20131, Aguascalientes, Ags. México, teléfono: 014499108536, correo electrónico: roal3micasa@aol.comAv. Universidad \#940 Ciudad Universitaria CP 20131, Aguascalientes, Ags. México, teléfono: 01 449 9108536, correo electrónico: roal3micasa@aol.com 


\section{Introducción}

Cáncer, es el término común para un grupo de enfermedades caracterizadas por el crecimiento y diseminación no controlada de células anormales, desplazando a las células sanas y utilizando los nutrientes requeridos por éstas ${ }^{1}$. La leucemia implica la proliferación no controlada de los leucocitos y sus precursores en los órganos formadores de sangre, con infiltración a otros órganos. Los síntomas y signos de leucemia son inespecíficos e incluyen: fatiga fácil, malestar generalizado, fiebre, palidez, hemorragia, pérdida de peso, dolor óseo o articular, esplenomegalia, hepatomegalia, anemia, náusea y vómito. ${ }^{2}$

Las leucemias se clasifican en agudas y crónicas según su evolución y en linfoides o mieloides según la línea celular alterada. La leucemia linfoide aguda (LLA) es una enfermedad de rápida progresión que es ocasionada por la acumulación de las células inmaduras, no funcionales en la médula ósea, de estirpe linfoide conocidas como linfoblastos. La médula ósea a menudo no puede producir suficientes granulocitos, eritrocitos y plaquetas, debido a la elevada proliferación de los linfoblastos y al desplazamiento de los precursores normales en la misma; por lo que aparecen anemia, infecciones frecuentes, equimosis y hemorragias ${ }^{3}$

La leucemia es la neoplasia más frecuente en la edad pediátrica, representando el $35 \%$ de todos los cánceres y a diferencia del adulto, el tipo más común en los niños es la leucemia linfoblástica aguda (LLA) constituyendo el 80 - $85 \%$ de todas las leucemias de este grupo de edad ${ }^{4}$

Una de las complicaciones que más frecuentemente se presentan en los pacientes con cáncer es la caquexia que significa "condición pobre". La caquexia en el paciente con cáncer es un síndrome metabólico complejo que involucra falla en la ingestión adecuada del alimento, utilización deficiente de nutrimentos y destrucción persistente de la masa de células corporales como respuesta del huésped o una reacción ante factores derivados del tumor. El metabolismo intermediario de hidratos de carbono, proteínas y grasas se ve afectado. Representa de un $10 \%$ a un $22 \%$ de todas las muertes por cáncer. Es un síndrome bifásico, que consiste en un "período preclínico" y "la fase de caquexia" 5

La nutrición en estos pacientes se ve afectada de forma negativa por diferentes factores: el propio cáncer, la modalidad del tratamiento, el estado nutricional y su estado general. ${ }^{2}$ La orientación alimentaria comprende una serie de actividades realizada por el equipo de nutrición especializado, donde se ve implicado la evaluación nutricia y la intervención, cuyo objetivo es proporcionar un apoyo nutricio eficaz y confiable a los niños con cáncer; con el fin de contrarrestar el consumo de sustratos endógenos y, por lo tanto, la falla nutricia causada tanto por el crecimiento del tumor como 
por los cambios metabólicos que induce en el huésped, así como, las complicaciones propias de la terapéutica antineoplásica. ${ }^{6}$

La evaluación nutricional ha sido definida como la interpretación de información obtenida a partir de parámetros dietéticos, bioquímicos, clínicos y antropométricos, se utiliza para determinar el estado de nutrición del paciente y para planear la apropiada intervención nutricional. El propósito de una evaluación nutricional en pacientes oncológicos es identificar si el sujeto está desnutrido o en riesgo de estarlo, si presenta complicaciones nutricionales por el sitio de tumor y por los tratamientos empleados (cirugía, radioterapia y quimioterapia). Además, la evaluación nutricia permite una adecuada monitorización del estado nutricional durante el tratamiento e incluso una vez finalizado el mismo, en el seguimiento de la evolución y si el paciente presenta secuelas relacionadas con la nutrición ${ }^{7}$

La intervención nutricional, que acompaña el tratamiento oncológico, se centra principalmente, en la optimización del balance entre el gasto energético y la ingesta. Para alcanzar objetivos específicos tales como: disminución de la tasa de complicaciones, mejorar la respuesta y la tolerancia al tratamiento oncológico. ${ }^{8}$ Algunos autores consideran que el tratamiento nutricional debe ser parte del soporte oncológico integral, porque contribuye considerablemente a la mejoría de la calidad de vida. ${ }^{9}$

Pietsch y cols realizaron un estudio sobre las medidas del estado nutricio en niños con cáncer, ellos reportan que se deben realizar medidas bioquímicas y antropométricas (altura, peso, la circunferencia media de brazo y la albúmina sérica). En este trabajo se comprobó que un soporte nutricio en los niños con cáncer depende del estado nutricio al diagnóstico, la intensidad de la terapia y la probabilidad de que exista toxicidad gastrointestinal significativa. También demostraron que la importancia del soporte nutricio radica en los resultados de efectos adversos relacionados con la desnutrición. Estos resultados incluyen disminución de la capacidad para tolerar la quimioterapia, incidencia en el incremento de infección y disminución de la tasa de sobrevivencia. La nutrición enteral o parenteral puede ser importante en tratamiento de estos niños. ${ }^{9}$

Los niños con leucemia linfoblástica aguda cursan con un catabolismo aumentado por lo que su estado nutricio se ve deteriorado. La nutrición es un proceso fundamental en el manejo de los pacientes con cáncer e influye de manera importante en la morbilidad como en la mortalidad. El plan alimentario es un instrumento que ayuda al paciente a una mejor evolución de la enfermedad previniendo el catabolismo aumentado ${ }^{10}$.

En el presente trabajo se pretende identificar de qué manera influye la aplicación de un plan alimentario en los niños con LLA, en comparación con los que no lo reciben y observar cómo influye en el cambio del índice de masa corporal (IMC). 


\section{Material y métodos}

El estudio se realizó en el Servicio de Oncología Pediátrica del Centenario Hospital Miguel Hidalgo (CHMH), en la ciudad de Aguascalientes, Ags, de septiembre a noviembre de 2010. La selección de los pacientes fue a través de un muestreo por conveniencia, para lo cual se estudiaron un total de 23 niños de dos a 15 años de edad con diagnóstico de leucemia linfoblástica aguda comprobado mediante aspirado de médula ósea y/o citometría de flujo. Fueron clasificados en riesgo habitual o alto, los cuales fueron sometidos a tratamiento con quimioterapia de inducción a la remisión con los protocolos vigentes para tal efecto en el $\mathrm{CHMH}$. La asignación de los pacientes fue aleatoria y cegada para los investigadores, conformándose dos grupos uno con asesoramiento nutricio y otro sin asesoramiento nutricio (12 y 11 pacientes respectivamente).

A los que recibieron orientación nutricia se les brindó un plan alimentario adecuado para sus necesidades individuales. Se realizó una evaluación del estado nutricio que tomaba en cuenta: ficha de identificación, antecedentes heredo familiares, antecedentes personales patológicos, antecedentes personales no patológicos, diagnóstico médico, evaluación antropométrica, evaluación clínica y test de variaciones en el apetito. Tomando en cuenta los datos referidos se establecieron el estado nutricio de los niños al igual que su requerimien- to calórico y en base a ellos al grupo con tratamiento se le asignó la dieta adecuada considerando sus requerimientos.

Los padres de estos niños recibieron un menú adecuado al requerimiento energético del niño, lista de equivalentes para dar mayor opción a la variación de la dieta (explicando el manejo del Sistema Mexicano de Equivalentes para su mejor comprensión y una adecuada utilización), recomendaciones para prevenir o disminuir complicaciones comunes en pacientes con cáncer como: fatiga, sequedad en boca, saliva espesa, úlceras bucales, anorexia, pérdida de peso, diarrea, estreñimiento, aversión a alimentos y sabores específicos, náuseas, disfagia, saciedad precoz y dieta correcta. Se prosiguió a realizar la historia clínica nutricia de cada niño y un test de cambios en el apetito que pueden presentarse a lo largo del tratamiento. Se obtuvo el peso y la talla de cada niño al iniciar el estudio, a la semana siguiente se entregó el plan nutricio a cada padre de familia y en el transcurso de la investigación se fue monitoreando el apego al plan en el primer grupo y cambios en el apetito, alteraciones del tracto digestivo y variaciones significativas en la dieta común en ambos grupos. Al final se volvió a tomar el peso y la talla. Para el análisis estadístico se obtuvieron medias, varianza y porcentajes, así mismo se utilizó prueba de $\mathrm{T}$ de Student para la comparación de medias, se consideró significancia con una $p<0.05$

| | | | | | | | | | | | | | | | | | | | | | | | | | | | | | | | | | | | | | | | | | | | | | | | | | | | | | | | | | | | | | | | | | | | | | | | | | | | | | | | | | | | | | | | | | | | | | | | | | | | | | | | | | | | | | | | | | | | | | |

\section{Resultados}

Se estudiaron 23 pacientes, el 30\% $(n=7)$ del sexo femenino y el $70 \%(n=16)$ del sexo maslculino. Al primer grupo $(n=12)$ se les brindó un plan alimentario (3/12 mujeres y $9 / 12$ hombres). Al segundo grupo $(n=11)$ no se les brindó un plan alimentario, y se consideraron controles, (4/11 mujeres y $7 / 11$ hombres). Se les midió el IMC antes y después de la intervención. (Ver ta- bla 1). En el grupo de pacientes con intervención alimentaria, el promedio de IMC inicial fue de $17.16 \pm 3.81 \mathrm{~kg} / \mathrm{m} 2$ y el final de $17.34 \pm 3.69 \mathrm{~kg} / \mathrm{m}^{2}$, por lo que el incremento observado fue de $0.18 \mathrm{~kg} / \mathrm{m} 2$ en un lapso de 2 meses (figura 1). De los 12 pacientes con plan alimentario, se observó un incremento en el IMC en 7 pacientes y en los otros 5 disminuyó (figura 3). 
En el segundo grupo, pacientes a los que no se brindó plan alimentario se obtuvo un IMC inicial de $16.87 \mathrm{~kg} / \mathrm{m} 2$ y final de $16.80 \mathrm{~kg} / \mathrm{m}^{2}$, que representa una disminución de $0.07 \mathrm{~kg} / \mathrm{m}^{2}$ (figura 2). De los 11 pacientes sin plan alimentario $(n=11)$ se observó que en 7 de los pacientes disminuyó su IMC y 4 tuvieron un aumento del IMC (Figura 4).

Al comparar el IMC inicial contra el final no encontramos diferencia significativa entre ambos valores $(p>0.05)$ (Tabla 1).

\section{Tabla I}

Comparación del IMC en dos grupos de pacientes con LLA con y sin apoyo con un plan alimentario en el Centenario Hospital Miguel Hidalgo de la Ciudad de Aguascalientes

\begin{tabular}{|l|lll|}
\hline & IMC inicial Grupo I $(\mathrm{n}=12)$ & IMC final Grupo I $(\mathrm{n}=12)$ & $\mathrm{p}$ \\
\hline $\mathrm{IMC}\left(\mathrm{kg} / \mathrm{m}^{2}\right)$ promedio \pm DS & $17.16 \pm 3.81$ & $17.34 \pm 3.69$ & 0.86 \\
\hline & & & \\
\hline & & & $\mathrm{P}$ \\
\hline $\mathrm{IMC}\left(\mathrm{kg} / \mathrm{m}^{2}\right)$ promedio \pm DS & $16.87 \pm 1.59$ & IMC final Grupo II $(\mathrm{n}=11)$ & 0.66 \\
\hline
\end{tabular}

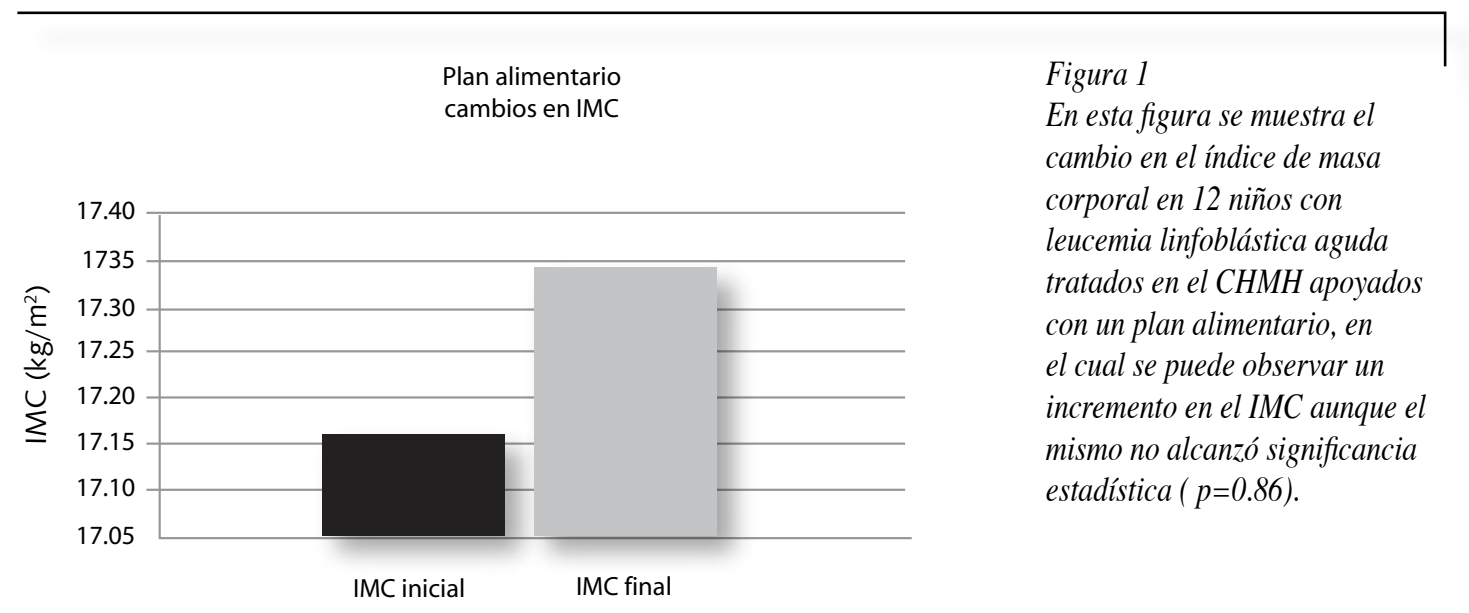

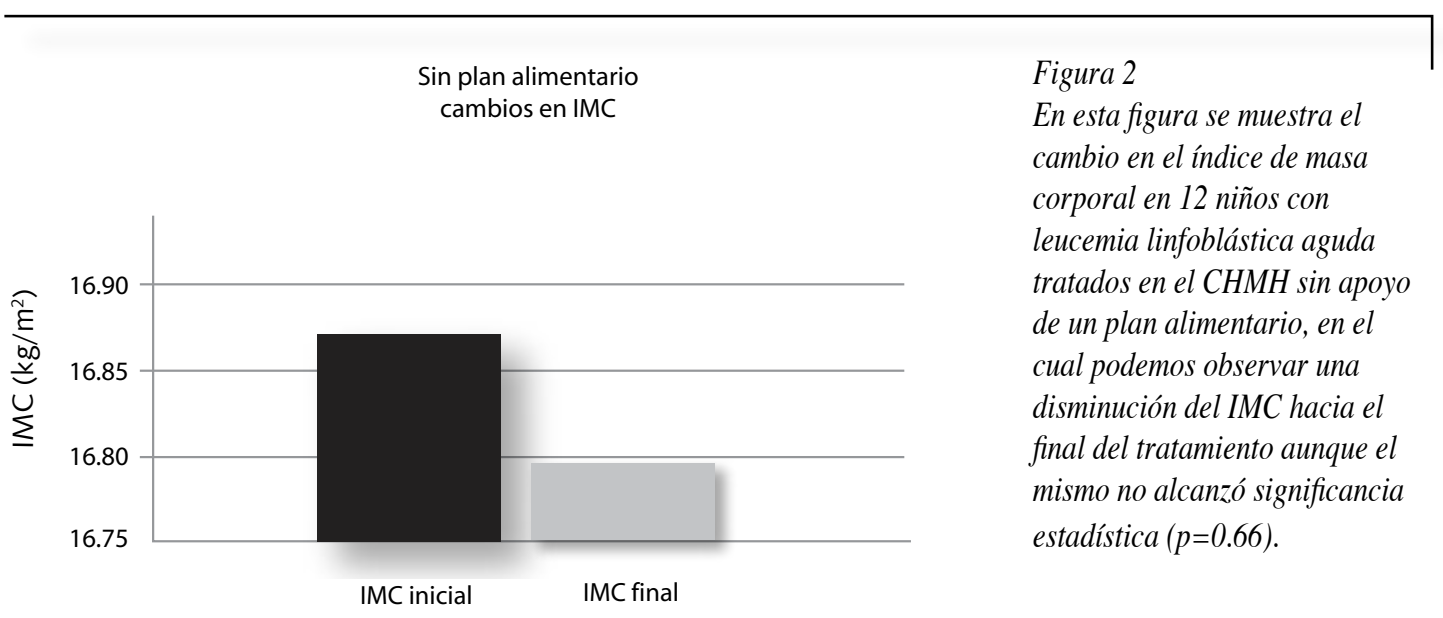




\section{Cambios IMC por niño plan alimentario}

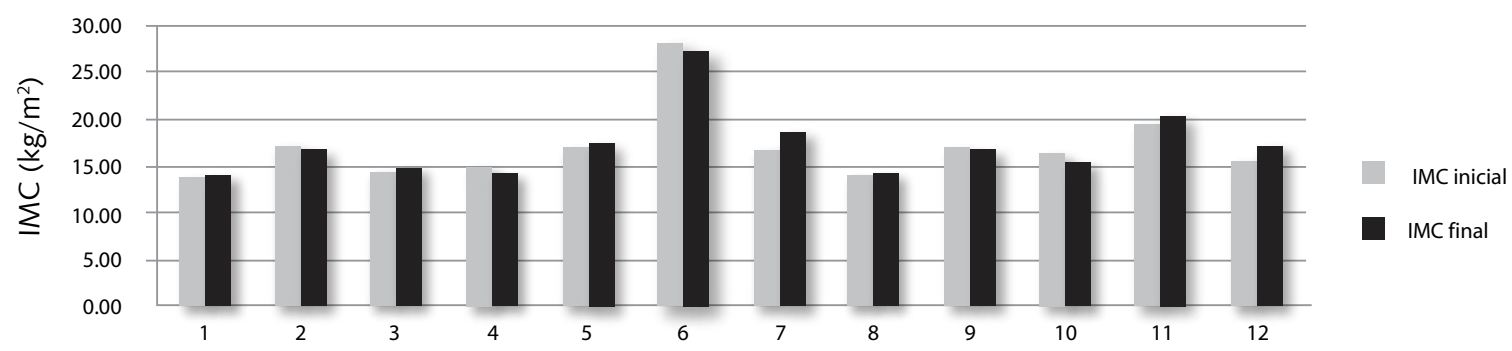

Figura 3

Cambio en el IMC por cada niño al que se le apoyó con plan alimentario, se puede observar que el IMC aumentó en siete niños y disminuyó en cinco.

Cambios IMC por niño

sin plan alimentario

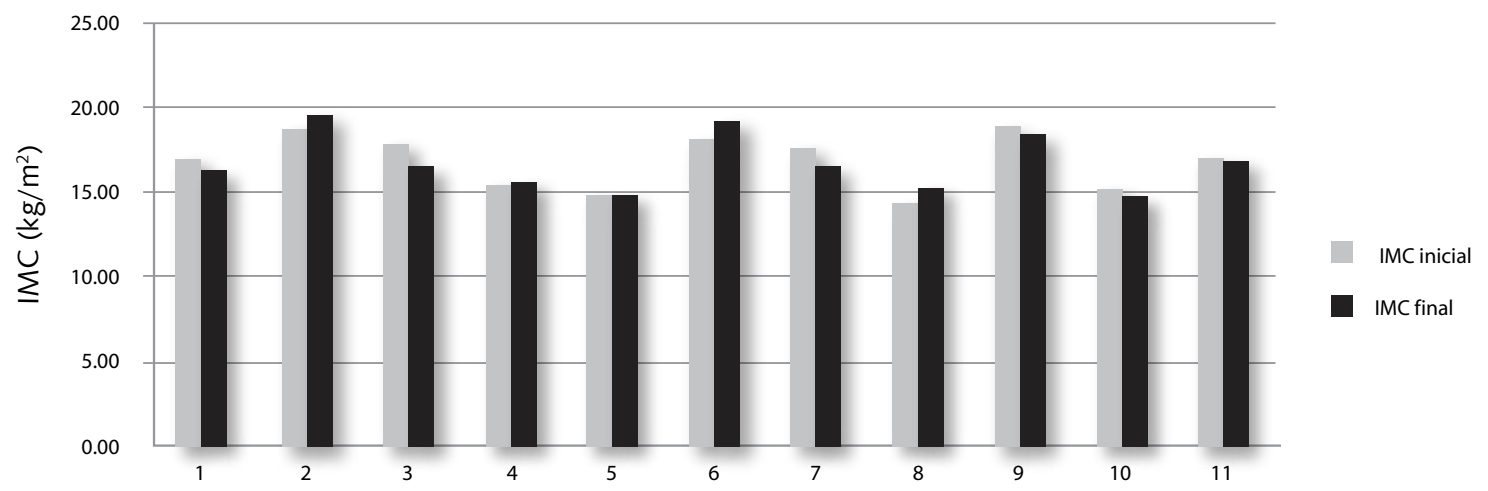

Figura 4

Cambio en el IMC por cada niño sin el apoyo de plan alimentario, se puede observar que el IMC disminuyó en siete niños y aumentó en cuatro.

\section{Discusión}

Los principales factores que modifican el peso y alteran el estado nutricio en los niños con cáncer, son el aumento en el requerimiento calórico debido al estrés provocado por la enfermedad, así como las complicaciones relacionadas al sistema digestivo que disminuyen la ingesta alimentaria como úlceras bucales, estreñimiento, diarrea, náusea, vómito, aversión a alimentos y sabores específicos o disfagia provocados por el tratamiento, aunado con los requerimientos propios de la edad en etapa infantil, todo lo cual favorece el desarrollo de un estado de caquexia, secundario al gran número de interacciones entre citoquinas proinflamatorias y el metabolismo del huésped y que da como resultado final 
el deterioro del estado nutricio del paciente. ${ }^{11-12}$

La intervención nutricional, que acompaña el tratamiento oncológico, se debe centrar principalmente en la optimización del balance entre el gasto energético y la ingesta; para alcanzar objetivos específicos tales como: disminución de la tasa de complicaciones, mejorar la respuesta y la tolerancia al tratamiento oncológico ${ }^{13}$

En el monitoreo realizado a los pacientes de ambos grupos se detectaron alteraciones en el sistema digestivo tales como úlceras bucales, estreñimiento, anorexia, náuseas y vómito las cuales fueron las principales variantes que provocaron disminución en el peso de nuestros niños estudiados. En el estudio realizado en pacientes con cáncer sin tratamiento nutricio de Gómez-Candela y Cols., ellos encontraron que el $44 \%$ de su población total presentó una pérdida de peso superior al $10 \%$ del peso ideal para la edad y la talla, en un lapso de 6 meses. En la figura 3 se puede observar que en cinco de los niños con tratamiento nutricio disminuyó su IMC y en la figura 4 se ob- serva que 7 niños sin tratamiento nutricio disminuyeron su IMC. La disminución del IMC evidencia pérdida de peso, lo cual se observa en mayor número en los pacientes sin tratamiento nutricio, aunque la diferencia entre ambos grupos no fue significativa quizá debido al tamaño de la muestra estudiada y a la elevada variabilidad del peso observado en nuestra población. ${ }^{10}$

En esta investigación se observó que las prácticas nutricionales óptimas pueden contribuir a mantener el peso y las reservas del organismo. En la primera medición se observó que el IMC de la población estudiada, a la cual se le había dado un plan nutricional, era menor que el de la población sin asignación de plan nutricional, esto era $17.16 \mathrm{~kg} / \mathrm{m}^{2}$ y $16.87 \mathrm{~kg} / \mathrm{m}^{2}$ respectivamente; al final del estudio el IMC del grupo con asesoría nutricia aumento a 17.34, lo que nos dice que se tuvo un mejor mantenimiento de las reservas corporales, dato contrario al IMC final del los pacientes sin asesoría nutricia, pues este disminuyó a 16.80 lo que significa un deterioro de las reservas del organismo.

\section{Conclusiones}

Una buena nutrición es fundamental para un niño que se encuentra bajo tratamiento del cáncer, por lo que un adecuado plan alimentario brinda la posibilidad de evitar o disminuir la pérdida de peso, controlar o revertir las complicaciones relacionadas con el sistema digestivo y consecuentemente lograr una mejor calidad de vida.

El estudio revela datos donde podemos observar que el IMC aumentó en aquellos pacientes a los cuales se les brindó un plan alimentario, al contrario de los pacientes que no recibieron el apoyo nutricio donde el IMC fue disminuyendo.

Si bien la diferencia en el IMC antes y después del apoyo nutricional no mostró significancia estadística, desde el punto de vista clínico, los resultados nos sugieren que el grupo con apoyo nutricional tuvo mejor mantenimiento de sus reservas corporales, dato contrario al IMC final de pacientes sin asesoría, el cual disminuyó $0.07 \mathrm{~kg} / \mathrm{m}^{2}$. Con este estudio proponemos que el plan alimentario puede contribuir a mantener las reservas corporales nutricionales. 


\section{Bibliografía}

1. Rosalinda T. Lagua, Virginia S. Claudio. Diccionario de nutrición y Dietoterapia (5ta Edición) México DF: Editorial Mc Graw Hill. pp. 43, 173

2. Stump S. (2002). Nutrición, Diagnóstico y tratamiento. ( $5^{\circ}$ edición). México: Editorial Mc Graw-Hill Interamericana. pp. 530-532

3. Fuentes $M$, Sánchez $C$, Granados M, Bóscan A. Evaluación del estado nutricional en niños con cáncer Revista Venezolana de Oncología. 2007;19(3):2049.

4. Google académico: http://www.spao.info/paginas/ multimenu2/pdf/0501.pdf. An Pediatr (Barc). [CDROM]. 2005;62:71-3

5. Robles-Gris J., Ochoa F. (1995). Apoyo nutricio en cáncer. Editorial Mc Graw Hill. 1ra edición. pp 37 41.

6. Calderón R. (2001), "Apoyo nutricio metabólico" Oncología médico quirúrgica pediátrica (1 ra edición), México, D.F.: Mc Graw Hill. Pp. 41-44

7. Sánchez Lara K, Turcott J, Sosa- Sánchez, GreenRenner Dan. Evaluación del estado de nutrición en pacientes con cáncer. Revista endocrinología y Nutrición. 2008;16: 165-171
8. Marín C, Laviano A, Pichard C, Gómez-Candela. Relación entre la intervención nutricional y la calidad de vida en el paciente con cáncer. Nutrición Hospitalaria: 2007 disponible en: http://scielo.isciii.es/pdf/ nh/v22n3/original5.pdf

9. Pietsch JB, Ford C. Niños con cáncer: Medidas del estado nutricio en el diagnóstico"

10. C. Gómez-Candela, L. M. Luengo, A. I. Cos, Valoración global subjetiva en el paciente neoplásico. Nutrición Hospitalaria: 2003;18(6) 353-357

11. Selwood K, Ward E, Gibson F. Assessment and management of nutritional challenges in children's cáncer care: a survey of current practice in the United Kingdom. Eur J Oncol Nurs. 2010;14(5):439-46

12. Viana $M B$, Fernandes $R A$, de Oliveira $B M$, Murao $M$, de Andrade Paes $C$, Duarte AA. Nutritional and socio-economic status in the prognosis of childhood acute lymphoblastic leukemia. Haematologica 2001;86(12):113-20.

13. Jaime-Pérez JC, González-Llano O, Herrera-Garza $J \mathrm{~L}$, Gutiérrez-Aguirre H, Vázquez-Garza E, GómezAlmaguer D. Assessment of nutritional status in children with acute lymphoblastic leukemia in Nothern México: A 5-year experience. Pediatr Blood cáncer. 2008:50(2 suppl):506-8 